\title{
HIGH ENERGY SCATTERING IN THE QUASI-POTENTIAL APPROACH
}

\author{
Nguyen Suan Han ${ }^{a, b}$ ], Le Hai Yen ${ }^{a}$, Nguyen Nhu Xuan ${ }^{c}$ \\ ${ }^{a}$ Department of Theoretical Physics, Vietnam National University, Hanoi, Vietnam. \\ ${ }^{b}$ The Abdus Salam International Centre for Theoretical Physics, Trieste, Italy. \\ ${ }^{c}$ Department of Theoretical Physics, Le Qui Don Technical University, Hanoi, Vietnam.
}

\begin{abstract}
Asymptotic behavior of the scattering amplitude for two scalar particles by scalar, vector and tensor exchanges at high energy and fixed momentum transfers is reconsidered in quantum field theory. In the framework of the quasi-potential approach and the modified perturbation theory a systematic scheme of finding the leading eikonal scattering amplitudes and its corrections are developed and constructed. The connection between the solutions obtained by quasi-potential and functional approaches is also discussed.The first correction to leading eikonal amplitude is found.
\end{abstract}

Keywords: Eikonal scattering theory, Quantum gravity.

\footnotetext{
${ }^{1}$ Email: lienbat76@gmail.com
} 


\section{Introduction}

The eikonal scattering amplitude for the high energy of the two particles in the limit of high energies and fixed momentum transfers is found by many authors in quantum field theory [1 -9$]$, including the quantum gravity $[9-20]$. Comparison of the results obtained by means of the different approaches for this problem has shown that they all coincide in the leading order approximation, while the corrections (non-leading terms) provided by them are rather different $[15,17,20,21,22,23]$. Determination of these corrections to gravitational scattering is now open problem $[10-14]$. These corrections play crucial role in such problems like strong gravitational forces near black hole, string modification of theory of gravity and other effects of quantum gravity $[9-20]$.

The purpose of the present paper is to develop a systematic scheme based on modified perturbation theory to find the correction terms to the leading eikonal amplitude for high-energy scattering by means of solving the Logunov-Tavkhelidze quasi-potential equation [24 - 27]. In spite of the lack of a clear relativistic covariance, the quasi-potential method keeps all information about properties of scattering amplitude which could be received starting from general principle of quantum field theory [25]. Therefore, at high energies one can investigate the analytical properties of the scattering amplitude, its asymptotic behavior and some regularities of a potential scattering etc. Exactly, as it has been done in the usual S-matrix theory [24]. The choice of this approach is dictated also by the following reasons: 1. in the framework of the quasi-potential approach the eikonal amplitude has a rigorous justification in quantum field theory [4]; 2. in the case of smooth potentials, it was shown that a relativistic quasi-potential and the Schrodinger equations lead to qualitatively identical results $[28,29]$.

The outline of the paper is as follows. In the second section the Logunov-Tavkhelidze quasipotential equation is written in an operator form. In the third section the solution of this equation is presented in an exponent form which is favorable to modify the perturbation theory. The asymptotic behavior scattering amplitude at high energies and fixed momentum transfers is considered in the fourth section. The lowest-order approximation of the modified theory is the leading eikonal scattering amplitude. Corrections to leading eikonal amplitude are also calculated. In the fifth section the solution of quasi-potential equation is presented in the form of a functional path integral. The connection between the solutions obtained by quasi-potential and functional integration is also discussed. It is shown that the approximations used are similar and the expressions for corrections to the leading eikonal amplitude are found identical. Finally, we draw our conclusions. 


\section{Two particle quasi-potential equation}

For simplicity, we shall first consider the elastic scattering of two scalar nucleons interacting with a scalar meson fields the model is described by the interaction Lagrangian $L_{i n t}=g \varphi^{2}(x) \phi(x)$. The results will be generalized to the case of scalar nucleons interacting with a neutral vector and graviton fields later. Following Ref.[23] for two scalar particle amplitude the quasi-potential equation with local quasi-potential has the form:

$$
T\left(\mathbf{p}, \mathbf{p}^{\prime} ; s\right)=g V\left(\mathbf{p}-\mathbf{p}^{\prime} ; s\right)+g \int d \mathbf{q} V(\mathbf{p}-\mathbf{q} ; s) K\left(\mathbf{q}^{2}, s\right) T\left(\mathbf{q}, \mathbf{p}^{\prime} ; s\right),
$$

where $K\left(\mathbf{q}^{2}, s\right)=\frac{1}{\sqrt{q^{2}+m^{2}}} \frac{1}{q^{2}+m^{2}-\frac{s}{4}-i \varepsilon}, s=4\left(\mathbf{p}^{2}+m^{2}\right)=4\left(\mathbf{p}^{\prime}+m^{2}\right)$ is the energy and $\mathbf{p}, \mathbf{p}^{\prime}$ and are the relative momenta of particles in the center of mass system in the initial and final states respectively. Equation (2.1) is one of the possible generalizations of the Lippman-Schwinger equation for the case of relativistic quantum field theory. The quasi-potential $V$ is a complex function of the energy and the relative momenta. The quasi-potential equation simplifies considerably if $V$ is a function of only the difference of the relative momenta and the total energy, i.e. if the quasi-potential is local 2. The existence of a local quasi-potential has been proved rigorously in the weak coupling case [27] and a method has been specified for constructing it. The local potential constructed in this manner gives a solution of Eq. (2.1), being equal to the physical amplitude on the mass shell $[24-26]$.

Making the following Fourier transformations

$$
\begin{gathered}
V\left(\mathbf{p}-\mathbf{p}^{\prime} ; s\right)=\frac{1}{(2 \pi)^{3}} \int d \mathbf{r} e^{i\left(\mathbf{p}-\mathbf{p}^{\prime}\right) \mathbf{r}} V(\mathbf{r} ; s), \\
T\left(\mathbf{p}, \mathbf{p}^{\prime} ; s\right)=\int d \mathbf{r} d \mathbf{r}^{\prime} e^{i\left(\mathbf{p r}-\mathbf{p}^{\prime} \mathbf{r}^{\prime}\right)} T\left(\mathbf{r}, \mathbf{r}^{\prime} ; s\right) .
\end{gathered}
$$

Substituting (2.2) and (2.3) into (2.1), we obtain

$$
\begin{gathered}
T\left(\mathbf{r}, \mathbf{r}^{\prime} ; \mathbf{s}\right)=\frac{\mathbf{g}}{(\mathbf{2} \pi)^{3}} \mathbf{V}(\mathbf{r} ; \mathbf{s}) \delta^{(3)}\left(\mathbf{r}-\mathbf{r}^{\prime}\right)+ \\
\frac{g}{(2 \pi)^{3}} \iint d \mathbf{q} \mathbf{K}\left(\mathbf{q}^{2} ; \mathbf{s}\right) \mathbf{V}(\mathbf{r} ; \mathbf{s}) \mathbf{e}^{-\mathbf{q r}} \int \mathbf{d} \mathbf{r}^{\prime \prime} \mathbf{e}^{\mathbf{i} \mathbf{q} \mathbf{r}^{\prime \prime}} \mathbf{T}\left(\mathbf{r}^{\prime \prime}, \mathbf{r}^{\prime} ; \mathbf{s}\right)
\end{gathered}
$$

and introducing the representation

$$
T\left(\mathbf{r}, \mathbf{r}^{\prime} ; s\right)=\frac{g}{(2 \pi)^{3}} V(\mathbf{r} ; s) F\left(\mathbf{r}, \mathbf{r}^{\prime} ; s\right)
$$

we obtain

$$
F\left(\mathbf{r}, \mathbf{r}^{\prime} ; s\right)=\delta^{(3)}\left(\mathbf{r}-\mathbf{r}^{\prime}\right)+\frac{g}{(2 \pi)^{3}} \int d \mathbf{q} K\left(\mathbf{q}^{2} ; s\right) e^{-i \mathbf{q r}} \times \int d \mathbf{r}^{\prime \prime} e^{i \mathbf{q} \mathbf{r}^{\prime \prime}} V\left(\mathbf{r}^{\prime \prime} ; s\right) F\left(\mathbf{r}^{\prime \prime}, \mathbf{r}^{\prime} ; s\right) .
$$

\footnotetext{
${ }^{2}$ Since the total energy $s$ appears as an external parameter of the equation, the term "local" here has direct meaning and it can appear in a three-dimensional $\delta$-function in the quasi-potential in the coordinate representation
} 
Defining the pseudo-differential operator

$$
\widehat{L_{r}}=K\left(-\nabla_{\mathbf{r}}^{2} ; s\right),
$$

then

$$
K(\mathbf{r} ; s)=\int d \mathbf{q} K\left(\mathbf{q}^{2} ; s\right) e^{-i \mathbf{q r}}=K\left(-\nabla_{r} ; s\right) \int d \mathbf{q} e^{-i \mathbf{q r}}=\widehat{L_{r}}(2 \pi)^{3} \delta^{(3)}(\mathbf{r}) .
$$

With allowance for (2.7) and (2.8), Eq. (2.6) can be rewritten in the symbolic form:

$$
F\left(\mathbf{r}, \mathbf{r}^{\prime} ; s\right)=\delta^{(3)}\left(\mathbf{r}-\mathbf{r}^{\prime}\right)+g \widehat{L_{r}}\left[V(\mathbf{r}, s) F\left(\mathbf{r}, \mathbf{r}^{\prime}, s\right)\right] .
$$

Eq. (2.8) is the Logunov-Tavkhelize quasi-potential equation in the operator form.

\section{$3 \quad$ Modified perturbation theory}

In the framework of the quasi-potential equation the potential is defined as an infinite power series in the coupling constant which corresponds to the perturbation expansion of the scattering amplitude on the mass shell. The approximate equation has been obtained only in the lowest order of the quasi-potential. Using this approximation the relativistic eikonal expression of elastic scattering amplitude was first found in quantum field theory for large energies and fixed momentum transfers [22]. In this paper we follow a somewhat different approach based on the idea of the modified perturbation theory proposed by Fradkin[30]. 3 The solution of equation (2.8) can be found in the form

$$
F\left(\mathbf{r}, \mathbf{r}^{\prime} ; s\right)=\frac{1}{(2 \pi)^{3}} \int d \mathbf{k} \exp [W(\mathbf{r} ; \mathbf{k} ; s)] e^{-i \mathbf{k}\left(\mathbf{r}-\mathbf{r}^{\prime}\right)} .
$$

Substituting (3.1) into (2.9) we have

$$
\begin{aligned}
& \exp W(\mathbf{r}, \mathbf{k} ; \mathbf{s})=1+g\left\{\widehat{L_{r}}[V(\mathbf{r} ; \mathbf{s}) \exp \mathbf{W}(\mathbf{r}, \mathbf{k} ; \mathbf{s})]\right. \\
& \left.\quad+V(\mathbf{r} ; \mathbf{s}) \exp \mathbf{W}(\mathbf{r}, \mathbf{k} ; \mathbf{s}) \mathbf{K}\left(\mathbf{k}^{\mathbf{2}} ; \mathbf{s}\right)\right\}
\end{aligned}
$$

Reducing this equation for the function $W(\mathbf{r} ; \mathbf{k} ; s)$, we get

$$
\exp W(\mathbf{r} ; \mathbf{k} ; s)=1+g \widehat{L_{r}}\{V(\mathbf{r}, s) \exp [W(\mathbf{r}, \mathbf{k} ; \mathbf{s})-\mathbf{i k r} \mathbf{r}]\} e^{i \mathbf{k r}} .
$$

The function $W(\mathbf{r} ; \mathbf{k} ; s)$ in exponent (3.1) can now be written as an expansion in series in the coupling constant g:

$$
W(\mathbf{r} ; \mathbf{k} ; s)=\sum_{n=1}^{\infty} g^{n} W_{n}(\mathbf{r} ; \mathbf{k} ; s) .
$$

\footnotetext{
${ }^{3}$ The interpretation of the perturbation theory from the view-point of the diagrammatic technique is as follows. The typical Feynman denominator of the standard perturbation theory is of the form $(A):\left(p+\sum q_{i}\right)^{2}+m^{2}-i \varepsilon=$ $p^{2}+m^{2}+2 p \sum q_{i}+\left(\sum q_{i}\right)^{2}$, where $p$ is the external momentum of the scalar (spinor) particle, and the $q_{i}$ are virtual momenta of radiation quanta. The lowest order approximation $(A)$ of modified theory is equivalent to summing all Feynman diagrams with the replacement: $\left(\sum q_{i}\right)^{2}=\sum\left(q_{i}\right)^{2}$ in each denominator $(A)$. The modified perturbation theory thus corresponds to a small correlation of the radiation quanta: $\mathbf{q}_{\mathbf{i}} \mathbf{q}_{\mathbf{j}}=\mathbf{0}$ and is often called the $\mathbf{q}_{\mathbf{i}} \mathbf{q}_{\mathbf{j}}$-approximation. In the framework of functional integration this approximation is called the straight-line path approximation i.e high-energy particles move along Feynman paths, which are practically rectilinear $[18,19]$.
} 
Substituting (3.4) into (3.3) and using Taylor expansion, the lhs.(3.3) is rewritten as follow

$$
1+\sum_{n=1}^{\infty} g^{n} W_{n}+\frac{1}{2 !}\left(\sum_{n=1}^{\infty} g^{n} W_{n}\right)^{2}+\frac{1}{3 !}\left(\sum_{n=1}^{\infty} g^{n} W_{n}\right)^{3}+\ldots,
$$

and the rhs.(3.3) has form

$$
\begin{aligned}
& 1+g\left\{\hat{L}_{r}\left[V(\mathbf{r} ; s)\left(1+\sum_{n=1}^{\infty} g^{n} W_{n}+\frac{1}{2 !}\left(\sum_{n=1}^{\infty} g^{n} W_{n}\right)^{2}+\frac{1}{3 !}\left(\sum_{n=1}^{\infty} g^{n} W_{n}\right)^{3}+\ldots\right)\right]+\right. \\
& \left.+V(\mathbf{r} ; s)\left[1+\sum_{n=1}^{\infty} g^{n} W_{n}+\frac{1}{2 !}\left(\sum_{n=1}^{\infty} g^{n} W_{n}\right)^{2}+\frac{1}{3 !}\left(\sum_{n=1}^{\infty} g^{n} W_{n}\right)^{3}+\ldots\right] K(\mathbf{k} ; s)\right\} .
\end{aligned}
$$

From (3.5) and (3.6), to compare with two sides of Eq.(3.3) following $g$ coupling, we derive the following expressions for the functions $W_{n}(\mathbf{r} ; \mathbf{k} ; s)$

$$
\begin{gathered}
W_{1}(\mathbf{r} ; \mathbf{k} ; s)=\int d \mathbf{q} V(\mathbf{q} ; s) K\left[(\mathbf{k}+\mathbf{q})^{2} ; s\right] e^{-i \mathbf{q r}} ; \\
W_{2}(\mathbf{r} ; \mathbf{k} ; s)=-\frac{W_{1}^{2}(\mathbf{r} ; \mathbf{k} ; s)}{2 !}+\frac{1}{2} \int d \mathbf{q}_{1} d \mathbf{q}_{2} V\left(\mathbf{q}_{1} ; s\right) V\left(\mathbf{q}_{2} ; s\right) K\left[\left(\mathbf{k}+\mathbf{q}_{1}+\mathbf{q}_{2}\right)^{2} ; s\right] \times \\
\times\left[K\left(\mathbf{k}+\mathbf{q}_{1} ; \mathbf{s}\right)+\mathbf{K}\left(\mathbf{k}+\mathbf{q}_{2} ; \mathbf{s}\right)\right] e^{-i \mathbf{q}_{1} \mathbf{r}-i \mathbf{q}_{2} \mathbf{r}} ; \\
W_{3}(\mathbf{r} ; \mathbf{k} ; s)=-\frac{W_{1}^{2}(\mathbf{r} ; \mathbf{k} ; s)}{3 !}+\int d \mathbf{q}_{1} d \mathbf{q}_{2} d \mathbf{q}_{3} V\left(\mathbf{q}_{1} ; s\right) V\left(\mathbf{q}_{2} ; s\right) V\left(\mathbf{q}_{3} ; s\right) K\left[\left(\mathbf{k}+\mathbf{q}_{1}\right)^{2} ; s\right] \\
\times K\left[\left(\mathbf{k}+\mathbf{q}_{1}+\mathbf{q}_{2}\right)^{2} ; s\right] K\left[\left(\mathbf{k}+\mathbf{q}_{1}+\mathbf{q}_{2}+\mathbf{q}_{3}\right)^{2} ; s\right] e^{-i\left(\mathbf{q}_{1}+\mathbf{q}_{2}+\mathbf{q}_{3}\right) \mathbf{r}} .
\end{gathered}
$$

Oversleeves by $W_{1}$ only we obtain from Eqs (3.1), (3.4) and (2.3) the approximate expression for the scattering amplitude [22]

$$
T_{1}\left(\mathbf{p}, \mathbf{p}^{\prime} ; s\right)=\frac{g}{(2 \pi)^{3}} \int d \mathbf{r} e^{i\left(\mathbf{p}-\mathbf{p}^{\prime}\right) \mathbf{r}} V(\mathbf{r}, s) e^{g W_{1}(\mathbf{r}, \mathbf{p}, s)} .
$$

To establish the meaning of this approximation, we expand $T_{1}$ in a series in $g$ :

$$
\begin{gathered}
T_{1}^{(n+1)}\left(\mathbf{p}, \mathbf{p}^{\prime} ; s\right)=\frac{g^{n+1}}{n !} \int d \mathbf{q}_{1} \ldots d \mathbf{q}_{n} V\left(\mathbf{q}_{1} ; s\right) \ldots V\left(\mathbf{q}_{n} ; s\right) \\
\times V\left(\mathbf{p}-\mathbf{p}^{\prime}-\sum_{i=1}^{n} q_{i} ; s\right) \prod_{i=0}^{n} K\left[\left(\mathbf{q}_{i}+\mathbf{p}^{\prime}\right)^{2} ; s\right] .
\end{gathered}
$$

Let us compare Eq. (3.10) with the $(n+1)-t h$ iteration term of exact Eq. (2.1)

$$
\begin{gathered}
T^{(n+1)}\left(\mathbf{p}, \mathbf{p}^{\prime} ; \mathbf{s}\right)=\int \mathbf{d} \mathbf{q}_{1} \ldots \mathbf{d q}_{\mathbf{n}} \mathbf{V}\left(\mathbf{q}_{1} ; \mathbf{s}\right) \ldots \mathbf{V}\left(\mathbf{q}_{\mathbf{n}} ; \mathbf{s}\right) \times \mathbf{V}\left(\mathbf{p}-\mathbf{p}^{\prime}-\sum_{\mathbf{i}=\mathbf{1}}^{\mathbf{n}} \mathbf{q}_{\mathbf{i}} ; \mathbf{s}\right) \\
\sum_{p} K\left[\left(\mathbf{q}_{1}+\mathbf{p}^{\prime}\right)^{2} ; s\right] K\left[\left(\mathbf{q}_{1}+\mathbf{q}_{2}+\mathbf{p}^{\prime}\right)^{2} ; s\right] \ldots K\left[\left(\sum_{i=1} \mathbf{q}_{i}+\mathbf{p}^{\prime}\right)^{2} ; s\right],
\end{gathered}
$$

where $\sum_{p}$ is the sum over the permutations of the momenta $\mathbf{p}_{1}, \mathbf{p}_{2} \ldots \mathbf{p}_{n}$. It is readily seen from (3.11) and (3.12) that our approximation in the case of the Lippmann-Schwinger equation is identical with the $\mathbf{q}_{i} \mathbf{q}_{j}$ approximation. 


\section{Asymptotic behavior of the scattering amplitude at high en- ergies}

In this section the solution of the Logunov-Tavkhelidze quasi-potential equation obtained in the

previous section for the scattering amplitude can be used to find the asymptotic behavior as $s \rightarrow \infty$ for fixed $t$. In the asymptotic expressions we shall retain both the principal term and the following term, using the formula

$$
e^{W\left(\mathbf{r}, \mathbf{p}^{\prime} ; s\right)}=e^{W_{1}\left(\mathbf{r}, \mathbf{p}^{\prime} ; s\right)}\left[1+g^{2} W_{2}\left(\mathbf{r}, \mathbf{p}^{\prime} ; s\right)+\ldots\right],
$$

where $W_{1}$ and $W_{2}$ are given by (3.7) and (3.8).

We take the $z$ axis along the vector $\left(\mathbf{p}+\mathbf{p}^{\prime}\right)$ then

$$
\mathbf{p}-\mathbf{p}^{\prime}=\boldsymbol{\Delta}_{\perp} ; \quad \boldsymbol{\Delta}_{\perp} \mathbf{n}_{\mathbf{z}}=0 ; \quad t=-\boldsymbol{\Delta}_{\perp}^{2} .
$$

Noting

$$
\begin{gathered}
K\left(\mathbf{p}+\mathbf{p}^{\prime} ; s\right)=\frac{1}{\sqrt{\left(\mathbf{p}+\mathbf{p}^{\prime}\right)^{2}+m^{2}}} \frac{1}{\left(\mathbf{p}+\mathbf{p}^{\prime}\right)^{2}-\frac{s}{4}+m^{2}-i \varepsilon} \mid \begin{array}{l}
s \rightarrow \infty \\
t-\text { fixed }
\end{array} \\
=\frac{2}{s\left(q_{z}^{2}-i \varepsilon\right)}\left[1-\frac{3 q_{z}^{2}+\mathbf{q}_{\perp}{ }^{2}+\mathbf{q}_{\perp} \triangle_{\perp}}{\sqrt{s}\left(q_{z}-i \epsilon\right)}\right]+O\left(\frac{1}{s^{2}}\right),
\end{gathered}
$$

and using Eqs (3.4), (3.7) and (3.8) we obtain

$$
\begin{gathered}
W_{1}=\left(\frac{W_{10}}{s}\right)+\left(\frac{W_{11}}{s \sqrt{s}}\right)+O\left(\frac{1}{s^{2}}\right) ; \\
W_{2}=\left(\frac{W_{20}}{s^{2} \sqrt{s}}\right)+O\left(\frac{1}{s^{3}}\right),
\end{gathered}
$$

where

$$
\begin{gathered}
W_{10}=2 \int d \mathbf{q} V(\mathbf{q} ; s) \frac{e^{i \mathbf{q r}}}{\left(q_{z}^{2}-i \varepsilon\right)^{2}}=2 i \int_{-\infty}^{z} d z^{\prime} V\left(\sqrt{\mathbf{q}_{\perp}^{2}+z^{\prime 2}} ; s\right) ; \\
W_{11}=-2 \int d \mathbf{q} \mathbf{V}(\mathbf{q} ; \mathbf{s}) \mathbf{e}^{-\mathbf{i} \mathbf{q r}} \frac{\mathbf{3 q}_{\mathbf{z}}^{\mathbf{2}}+\mathbf{q}_{\perp}^{\mathbf{2}}+\mathbf{q}_{\perp} \triangle_{\perp}}{\left(\mathbf{q}_{\mathbf{z}}-\mathbf{i} \epsilon\right)^{\mathbf{2}}} \\
=-6 V\left(\sqrt{\mathbf{q}_{\perp}^{\mathbf{2}}+\mathbf{z}^{\prime \mathbf{2}}} ; s\right)+2\left(-\nabla_{\perp}^{\mathbf{2}}-\mathbf{i} \mathbf{q}_{\perp} \nabla_{\perp}\right) \times \int_{-\infty}^{\mathbf{z}} \mathbf{d \mathbf { z } ^ { \prime }} \mathbf{V}\left(\sqrt{\mathbf{q}_{\perp}^{\mathbf{2}}+\mathbf{z}^{\prime \mathbf{2}}} ; \mathbf{s}\right) ; \\
W_{20}=-4 \int d \mathbf{q}_{\mathbf{1}} \mathbf{d} \mathbf{q}_{\mathbf{2}} \mathbf{e}^{-\mathbf{i}\left(\mathbf{q}_{1}+\mathbf{q}_{2}\right) \mathbf{r}} \mathbf{V}\left(\mathbf{q}_{\mathbf{1}} ; \mathbf{s}\right) \mathbf{V}\left(\mathbf{q}_{\mathbf{2}} ; \mathbf{s}\right) \\
\times \frac{3 q_{1 z} q_{2 z}+\mathbf{q}_{1} \perp \mathbf{q}_{\mathbf{2}} \perp}{\left(q_{1 z}-i \varepsilon\right)\left(q_{2 z}-i \varepsilon\right)\left(q_{1 z}+q_{2 z}-i \varepsilon\right)}
\end{gathered}
$$




$$
=-4 i\left\{3 \int_{-\infty}^{z} d z^{\prime} V^{2}\left(\sqrt{\mathbf{q}_{\perp}^{\mathbf{2}}+\mathbf{z}^{\prime \mathbf{2}}} ; s\right)+\left[\nabla_{\perp} \int_{-\infty}^{z^{\prime}} d z^{\prime \prime} V^{2}\left(\sqrt{\mathbf{q}_{\perp}^{2}+z^{\prime \prime 2}} ; s\right)\right]^{2}\right\} .
$$

In the limit $s \rightarrow \infty$ and $(t / s) \rightarrow 0 W_{10}$ makes the main contribution, and the remaining terms are corrections. Therefore, the function $\exp W$ can be represented by means of the expansion (4.1) where $W_{10}, W_{11}$ and $W_{20}$ are determined by Eqs. (4.6) - (4.8) respectively. The asymptotic behavior scattering amplitude can be written in the following form

$$
\begin{gathered}
T\left(\mathbf{p}, \mathbf{p}^{\prime} ; \mathbf{s}\right)=\frac{\mathbf{g}}{(\mathbf{2} \pi)^{\mathbf{3}}} \int \mathbf{d}^{2} \mathbf{r}_{\perp} \mathbf{d z} \mathbf{e}^{\mathbf{i} \boldsymbol{\Delta}_{\perp} \mathbf{r}_{\perp}} \mathbf{V}\left(\sqrt{\mathbf{r}^{\mathbf{2}}+\mathbf{z}^{\mathbf{2}}} ; \mathbf{s}\right) \\
\times \exp \left(g \frac{W_{10}}{s}\right)\left(1+g \frac{W_{11}}{s \sqrt{s}}+g^{2} \frac{W_{20}}{s^{2} \sqrt{s}}+\ldots\right) .
\end{gathered}
$$

Substituting (4.6) - (4.8) into (4.9) and making calculations, at high energy $s \rightarrow \infty$ and fixed momentum transfers $(t / s) \rightarrow 0$, we finally obtain[22]

$$
\begin{aligned}
& T(s, t)=\frac{g}{2 i(2 \pi)^{3}} \int d^{2} \mathbf{r}_{\perp} \mathbf{e}^{\mathbf{i} \boldsymbol{\Delta}_{\perp} \mathbf{r}_{\perp}} \times\left\{\mathbf{e}^{\left[\frac{2 \mathbf{i g}}{\mathbf{s}} \int_{-\infty}^{\infty} \mathbf{d z V}\left(\sqrt{\mathbf{r}_{\perp}^{2}+\mathbf{z}^{2}} ; \mathbf{s}\right)\right]}-\mathbf{1}\right\} \\
& -\frac{6 g^{2}}{(2 \pi)^{3} s \sqrt{s}} \int d^{2} \mathbf{r}_{\perp} \mathbf{e}^{\mathbf{i} \boldsymbol{\Delta}_{\perp} \mathbf{r}_{\perp}} \times \exp \left[\frac{\mathbf{2 i g}}{\mathbf{s}} \int_{-\infty}^{\infty} \mathbf{d z}^{\prime} \mathbf{V}\left(\sqrt{\mathbf{r}_{\perp}^{2}+\mathbf{z}^{\mathbf{2}}} ; \mathbf{s}\right)\right] \\
& \times \int_{-\infty}^{\infty} d z V\left(\sqrt{\mathbf{r}_{\perp}^{\mathbf{2}}+\mathbf{z}^{\mathbf{2}}} ; s\right)-\frac{i g}{(2 \pi)^{3} \sqrt{s}} \int d^{2} \mathbf{r}_{\perp} \mathbf{e}^{\mathbf{i} \boldsymbol{\Delta}_{\perp} \mathbf{r}_{\perp} \times} \\
& \int_{-\infty}^{\infty} d z\left\{\exp \left[\frac{2 i g}{s} \int_{z}^{\infty} d z^{\prime} V\left(\sqrt{\mathbf{r}_{\perp}^{\mathbf{2}}+\mathbf{z}^{\prime \mathbf{2}}} ; s\right)\right]-\exp \left[\frac{2 i g}{s} \int_{-\infty}^{\infty} d z^{\prime} V\left(\sqrt{\mathbf{r}_{\perp}^{\mathbf{2}}+\mathbf{z}^{\prime \mathbf{2}}} ; s\right)\right]\right\} \\
& \times\left\{\int_{z}^{\infty} d z^{\prime} \nabla_{\perp}^{2} \mathbf{V}\left(\sqrt{\mathbf{r}_{\perp}^{2}+\mathbf{z}^{\prime 2}} ; \mathbf{s}\right)-\frac{2 \mathbf{i g}}{\mathbf{s}}\left[\int_{\mathbf{z}}^{\infty} \mathbf{d z}^{\prime} \nabla_{\perp} \mathbf{V}\left(\sqrt{\mathbf{r}_{\perp}^{2}+\mathbf{z}^{2}} ; \mathbf{s}\right)\right]^{2}\right\} \\
& \left.-\frac{2 i g}{(2 \pi)^{3} s} \boldsymbol{\Delta}_{\perp}^{\mathbf{2}} \int \mathbf{d}^{2} \mathbf{r}_{\perp} \mathbf{V}\left(\sqrt{\mathbf{r}_{\perp}^{\mathbf{2}}+\mathbf{z}^{\mathbf{2}}} ; \mathbf{s}\right)\right] \mathrm{e}^{\mathrm{i} \boldsymbol{\Delta}_{\perp} \mathbf{r}_{\perp} \times} \\
& \int_{-\infty}^{\infty} z d z V\left(\sqrt{\mathbf{r}_{\perp}^{\mathbf{2}}+\mathbf{z}^{\mathbf{2}}} ; s\right) \exp \left[\frac{2 i g}{s} \int_{z}^{\infty} d z^{\prime} V\left(\sqrt{\mathbf{r}_{\perp}^{\mathbf{2}}+\mathbf{z}^{\prime \mathbf{2}}} ; s\right)\right] .
\end{aligned}
$$

In this expression (4.10) the first term describes the leading eikonal behavior of the scattering amplitude, while the remaining terms determine the corrections of relative magnitude $1 / \sqrt{s}$. The similar result Eq.(4.10) is also found by means of the functional integration [20].

As is well known from the investigation of the scattering amplitude in the Feynman diagrammatic technique, the high-energy asymptotic behavior can contain only logarithms and integral powers of $s$. A similar effect is observed here, since integration of the expression (4.10) leads to the vanishing of the coefficients for half-integral powers of $s$. Nevertheless, allowance for the terms that contain the half-integral powers of $s$ is needed for the calculations of the next corrections in the scattering amplitude, and leads to the appearance of the so-called retardation effects, which 
are absent in the principal asymptotic term.

In the limit of high energies $s \rightarrow \infty$ and for fixed momentum transfers $t$ the expression for the scattering amplitude within the framework of the functional - integration method takes the Glauber form with eikonal function corresponding to a Yukawa interaction potential between "nucleons". Therefore, the local quasi-potential for the interaction between the "nucleons" from perturbation theory in that region can be chosen by following forms. For the scalar meson exchange the quasi-potential decreases with energy

$$
V(r ; s)=-\frac{g^{2}}{8 \pi s} \frac{e^{-\mu r}}{r} .
$$

The first term in the expression (4.10) describes the leading eikonal behavior of the scattering amplitude. Using integrals calculated in the Appendix, we find

$$
\begin{gathered}
T_{\text {Scalar }}^{(0)}(s, t)=-\frac{g}{2 i(2 \pi)^{3}} \int d^{2} \mathbf{r}_{\perp} \mathbf{e}^{\mathbf{i} \boldsymbol{\Delta}_{\perp} \mathbf{r}_{\perp} \times} \\
\left\{\exp \left[\frac{2 i g}{s} \int_{-\infty}^{+\infty} d z V\left(\sqrt{\mathbf{r}_{\perp}^{\mathbf{2}}+\mathbf{z}^{\mathbf{2}}} ; s\right)\right]-1\right\} \\
=\frac{g^{4}}{4(2 \pi)^{4} s^{2}}\left[\frac{1}{\mu^{2}-t}-\frac{g^{3}}{8(2 \pi)^{2} s^{2}} F_{1}(t)+\frac{g^{6}}{48(2 \pi)^{5} s^{4}} F_{2}(t)\right] .
\end{gathered}
$$

The next term in (4.10) describes first correction to the leading eikonal amplitude

$$
\begin{gathered}
T_{\text {Scalar }}^{(1)}(s, t)=-\frac{6 g^{2}}{(2 \pi)^{3} s \sqrt{s}} \int d^{2} r_{\perp} e^{i \Delta_{\perp} r_{\perp}} \times \\
\exp \left[\frac{2 i g}{s} \int_{-\infty}^{+\infty} d z V\left(\sqrt{\mathbf{r}_{\perp}^{2}+\mathbf{z}^{\mathbf{2}}} ; s\right)\right] \times \int_{-\infty}^{+\infty} d z V\left(\sqrt{\mathbf{r}_{\perp}^{2}+z^{2}} ; s\right) \\
=\frac{3 g^{4}}{4(2 \pi)^{6} s^{2} \sqrt{s}}\left[\frac{2}{\mu^{2}-t}-\frac{g^{3}}{2(2 \pi)^{2} s^{2}} F_{1}(t)+\frac{g^{6}}{8(2 \pi)^{5} s^{4}} F_{2}(t)\right]
\end{gathered}
$$

where

$$
F_{1}(t)=\frac{1}{t \sqrt{1-\frac{4 \mu^{2}}{t}}} \ln \left|\frac{1-\sqrt{1-4 \mu^{2} / t}}{1+\sqrt{1-4 \mu^{2} / t}}\right|
$$

and

$$
F_{2}(t)=\int_{0}^{1} d y \frac{1}{\left(t y+\mu^{2}\right)(y-1)} \ln \left|\frac{\mu^{2}}{y\left(t y+\mu^{2}-t\right)}\right|
$$

A similar calculations can be applied for other exchanges with different spins. In the case of the vector model $L_{i n t}=-g \varphi^{\star} i \partial_{\sigma} \varphi A^{\sigma}+g^{2} A_{\sigma} A^{\sigma} \varphi \varphi^{\star}$ the quasi-potential is independent of energy

$$
V(r ; s)=-\frac{g^{2}}{4 \pi} \frac{e^{-\mu r}}{r}
$$

we find 


$$
\begin{aligned}
& T_{\text {Vector }}^{(0)}(s, t)=\frac{g^{4}}{2(2 \pi)^{4} s} \times\left[\frac{1}{\mu^{2}-t}-\frac{g^{3}}{4(2 \pi)^{2} s} F_{1}(t)+\frac{g^{6}}{12(2 \pi)^{5} s^{2}} F_{2}(t)\right] \\
& T_{\text {Vector }}^{(1)}(s, t)=\frac{3 g^{4}}{2(2 \pi)^{6} s \sqrt{s}} \times\left[\frac{2}{\mu^{2}-t}-\frac{g^{3}}{(2 \pi)^{2} s} F_{1}(t)+\frac{g^{6}}{2(2 \pi)^{5} s^{2}} F_{2}(t)\right]
\end{aligned}
$$

In the case of tensor model 4 , the quasi- potential increases with energy $V(r ; s)=\left(\kappa^{2} s / 2 \pi\right)\left(e^{-\mu r} / r\right)$, we have

$$
\begin{aligned}
& T_{\text {Tensor }}^{(0)}(s, t)=-\frac{\kappa^{4}}{(2 \pi)^{4}} \times\left[\frac{1}{\mu^{2}-t}+\frac{\kappa^{3}}{2(2 \pi)^{2}} F_{1}(t)+\frac{\kappa^{6}}{3(2 \pi)^{5}} F_{2}(t)\right] \\
& T_{\text {Tensor }}^{(1)}(s, t)=-\frac{3 \kappa^{4}}{(2 \pi)^{6} \sqrt{s}} \times\left[\frac{2}{\mu^{2}-t}+\frac{2 \kappa^{3}}{(2 \pi)^{2}} F_{1}(t)+\frac{2 \kappa^{6}}{(2 \pi)^{5}} F_{2}(t)\right]
\end{aligned}
$$

To conclude this section it is important to note that in the framework of standard field theory for the high-energy scattering, different methods have been developed to investigate the asymptotic behavior of individual Feynman diagrams and their subsequent summation. In different theories including quantum gravity the calculations of Feynman diagrams in the eikonal approximation is proceed in a similar way as analogous the calculations in QED. Reliability of the eikonal approximation depends on spin of the exchanges field $[5,6]$. The eikonal captures the leading behavior of each order in perturbation theory, but the sum of leading terms is subdominant to the terms neglected by this approximation. The reliability of the eikonal amplitude for gravity is uncertain [14]. Instead of the diagram technique perturbation theory, our approach is based on the exact expression of the scattering amplitude and modified perturbation theory which in lowest order contains the leading eikonal amplitude and the next orders are its corrections.

\section{Relationship between the operator and Feynman path meth- ods}

What actual physical picture may correspond to our result given by Eq. (4.10) ? To answer this question we establish the relationship between the operator and Feynman path methods in Ref. [31], which treats the quasi-potential equation in the language of functional integrals. The solution of this equation can be written in the symbolic form:

\footnotetext{
${ }^{4}$ The model of interaction of a scalar "nucleons" field $\varphi(x)$ with a gravitational field $g_{\mu \nu}(x)$ in the linear approximation to $h^{\mu \nu}(x) ;[18] L(x)=L_{0, \varphi}(x)+L_{0, \text { grav }}(x)+L_{\text {int }}(x)$, where

$$
\begin{gathered}
L_{0}(x)=\frac{1}{2}\left[\partial^{\mu} \varphi(x) \partial_{\mu} \varphi(x)-m^{2} \varphi^{2}(x)\right] \\
L_{i n t}(x)=-\frac{\kappa}{2} h^{\mu \nu}(x) T_{\mu \nu}(x), \\
T_{\mu \nu}(x)=\partial_{\mu} \varphi(x) \partial_{\nu} \varphi(x)-\frac{1}{2} \eta_{\mu \nu}\left[\partial^{\sigma} \varphi(x) \partial_{\sigma} \varphi(x)-m^{2} \varphi^{2}(x)\right],
\end{gathered}
$$

$T_{\mu \nu}(x)$-the energy momentum tensor of the scalar field. The coupling constant $\kappa$ is related to Newton's constant of gravitation $G$ by $\kappa^{2}=16 \pi G$
} 


$$
\begin{gathered}
\exp (W)=\frac{1}{1-g K\left[(-i \nabla-\mathbf{k})^{2}\right] V(\mathbf{r})} \times \mathbf{1} \\
=-i \int_{0}^{\infty} d \tau \exp [i \tau(1+i \varepsilon)] \times \exp \left\{-i \tau g K\left[(-i \nabla-\mathbf{k})^{2}\right] V(\mathbf{r})\right\} \times \mathbf{1} .
\end{gathered}
$$

In accordance with the Feynman parametrization [31], we introduce an ordering index $\eta$ and write Eq. (5.1) in the form

$$
\exp (W)=-i \int_{0}^{\infty} d \tau e^{i \tau(1+i \varepsilon)} \times \exp \left\{-i g \int_{0}^{\infty} d \eta K\left[\left(-i \nabla_{\eta+\varepsilon}-\mathbf{k}\right)^{2}\right] U\left(\mathbf{r}_{\eta}\right)\right\} \times \mathbf{1} .
$$

Using Feynman transformation

$$
F[P(\eta)]=\int D \mathbf{p} \int_{x(0)=0} \frac{D \mathbf{x}}{(2 \pi)^{3}} \times \exp \left\{i \int_{0}^{\tau} d \eta \dot{\mathbf{r}}(\eta)[\mathbf{p}(\eta)-P(\eta)]\right\} F[\mathbf{p}(\eta)],
$$

we write the solution of Eq. (2.8) in the form of the functional integral

$$
\begin{aligned}
& \exp (W)=-i \int_{0}^{\infty} d \tau e^{i \tau(1+i \varepsilon)} \int D \mathbf{p} \int_{x(0)=0} \frac{D \mathbf{x}}{(2 \pi)^{3}} \\
& \times \exp \left\{i \int_{0}^{\tau} d \eta \dot{\mathbf{x}}(\eta)[\mathbf{p}(\eta)-P(\eta)]\right\} G(\mathbf{x}, \mathbf{p} ; \tau) \times \mathbf{1} .
\end{aligned}
$$

In Eq.(5.4) we enter the function $G$

$$
G(\mathbf{x}, \mathbf{p} ; \tau)=\exp \left\{-\mathbf{i} \int_{\mathbf{0}}^{\tau} \mathbf{d} \eta \dot{\mathbf{x}}(\eta) \nabla_{\eta+\varepsilon}\right\} \times \exp \left\{-\mathbf{i g} \int_{\mathbf{0}}^{\tau} \mathbf{d} \eta \mathbf{K}\left[(\mathbf{p}(\eta)-\mathbf{k})^{\mathbf{2}}\right] \mathbf{V}\left(\mathbf{r}_{\eta}\right)\right\},
$$

which satisfies the equation

$$
\frac{d G}{d \tau}=\left\{-i g K\left[(\mathbf{p}(\tau)-\mathbf{k})^{2}\right] V(\mathbf{r}-\dot{\mathbf{x}}(\tau-\varepsilon)) \nabla\right\} G ; \quad G(\tau=0)=1 .
$$

Finding from Eq. (5.6) the operator function $G$ and substituting it into Eq. (5.6) for $W$ we obtained the following final expression:

$$
\begin{gathered}
\exp (W)=-i \int_{0}^{\infty} d \tau e^{i \tau(1+i \varepsilon)} \int D \mathbf{p} \frac{1}{(2 \pi)^{3}} \\
\times \int_{x(0)=0} \frac{D \mathbf{x}}{(2 \pi)^{3}} \exp \left\{i \int_{0}^{\tau} d \eta \dot{\mathbf{x}}(\eta) p(\eta)\right\} \exp \left(g \prod\right),
\end{gathered}
$$

where

$$
\begin{gathered}
\prod=-i \int_{0}^{\infty} d \tau K\left[(\mathbf{p}(\eta)-\mathbf{k})^{2}\right] \times V\left[\mathbf{r}-\int_{0}^{\tau} d \xi \mathbf{x}(\xi) \vartheta(\xi-\eta+\varepsilon)\right] \\
\prod^{2}=-\int_{0}^{\tau_{1}} \int_{0}^{\tau_{2}} d \tau_{1} d \tau_{2} K\left[\left(\mathbf{p}\left(\eta_{1}\right)-\mathbf{k}\right)^{2}\right] K\left[\left(\mathbf{p}\left(\eta_{2}\right)-(\mathbf{k})\right)^{2}\right]
\end{gathered}
$$




$$
\times V\left[\mathbf{r}_{1}-\int_{0}^{\tau_{1}} d \xi \mathbf{x}(\xi) \vartheta(\xi-\eta+\varepsilon)\right] \times V\left[\mathbf{r}_{2}-\int_{0}^{\tau_{2}} d \dot{x} \dot{x}(\xi) \vartheta(\xi-\eta+\varepsilon)\right] .
$$

Writing out the expansion $[2,3]$

$$
\exp (W)=\overline{\exp \left(g \prod\right)}=\exp \left(g \bar{\prod}\right) \sum_{n=0}^{\infty} \frac{g^{n}}{n !} \overline{\left(\prod-\bar{\prod}\right)^{n}}
$$

in which the sign of averaging denoted integration with respect to $\tau, \mathbf{x}(\eta)$ and $\mathbf{p}(\eta)$ with the corresponding measure ( see, for example Eq. (5.7) ), and performing the calculations, we find

$$
W_{1}=\bar{\Pi}, W_{2}=\frac{{\overline{\Pi^{2}}}^{2}-\bar{\Pi}^{2}}{2 !}, W_{3}=\frac{1}{3 !}\left[{\overline{\Pi^{3}}}^{3}-\bar{\Pi}^{3}-3 \bar{\Pi}\left(\bar{\Pi}^{2}-\bar{\Pi}^{2}\right)\right], \text { etc. }
$$

i.e. the expressions (5.10) and (4.1) are identical.

$$
\begin{aligned}
& W_{1}=\bar{\prod}=-i \int_{0}^{\infty} d \tau K\left[(\mathbf{p}(\eta)-\mathbf{k})^{\mathbf{2}}\right] \times \exp \left[-\int_{\mathbf{0}}^{\tau} \mathbf{d} \xi \mathbf{x}(\xi) \vartheta(\xi-\eta+\varepsilon) \nabla_{\eta}\right] \mathbf{V}(\tilde{\mathbf{r}}) \\
& =\int d \mathbf{q} e^{-\mathbf{q r}} K\left[(\mathbf{q}+\mathbf{k})^{2}\right] V(\mathbf{q} ; s) \\
& \overline{\prod^{2}}=K\left[\left(\nabla_{\mathbf{r}_{1}}+\nabla_{\mathbf{r}_{2}}+\mathbf{k}\right)^{\mathbf{2}}\right] \mathbf{K}\left[\left(\nabla_{\mathbf{r}_{1}}+\mathbf{k}\right)^{\mathbf{2}}\right] \times \mathbf{K}\left[\left(\nabla_{\mathbf{r}_{2}}+\mathbf{k}\right)^{2}\right] \mathbf{V}\left(\mathbf{r}_{1} ; \mathbf{s}\right) \mathbf{V}\left(\mathbf{r}_{2} ; \mathbf{s}\right) \\
& =\int d \mathbf{q}_{1} \int d \mathbf{q}_{2} e^{-i\left(\mathbf{q}_{1}+\mathbf{q}_{2}\right) \mathbf{r}} K\left[\left(\mathbf{q}_{1}+\mathbf{q}_{2}+\mathbf{k}\right)^{2}\right] \\
& \times\left\{K\left[\left(\mathbf{q}_{\mathbf{1}}+\mathbf{k}\right)^{2}\right]+K\left[\left(\mathbf{q}_{2}+\mathbf{k}\right)^{2}\right]\right\} V\left(\mathbf{r}_{1} ; s\right) V\left(\mathbf{r}_{\mathbf{2}} ; s\right) ; \\
& W_{2}=-\frac{W_{1}^{2}}{2 !}+\frac{1}{2} \int d \mathbf{q}_{\mathbf{1}} d \mathbf{q}_{\mathbf{2}} V\left(\mathbf{q}_{\mathbf{1}}\right) V\left(\mathbf{q}_{\mathbf{2}}\right) \times\left\{K\left[\left(\mathbf{q}_{\mathbf{1}}+\mathbf{k}\right)^{2} ; s\right]+K\left[\left(\mathbf{q}_{\mathbf{2}}+\mathbf{k}\right)^{2} ; s\right]\right\} ; \\
& W_{3}=-\frac{W_{1}^{3}}{3 !}+\int d \mathbf{q}_{1} d \mathbf{q}_{2} d \mathbf{q}_{3} V\left(\mathbf{q}_{1} ; s\right) V\left(\mathbf{q}_{2} ; s\right) V\left(\mathbf{q}_{3} ; s\right) \\
& \times K\left[\left(\mathbf{k}+\mathbf{q}_{1}\right)^{2} ; \mathbf{s}\right] \mathbf{K}\left[\left(\mathbf{k}+\mathbf{q}_{1}+\mathbf{q}_{2}\right)^{2} ; \mathbf{s}\right] \mathbf{K}\left[\left(\mathbf{k}+\mathbf{q}_{1}+\mathbf{q}_{2}+\mathbf{q}_{3}\right)^{2} ; \mathbf{s}\right] \\
& \times e^{-i\left(\mathbf{q}_{1}+\mathbf{q}_{2}+\mathbf{q}_{3}\right) \mathbf{r}} ; \text { etc }
\end{aligned}
$$

Restricting ourselves in the expansion (5.10) to the first term $(n=0)$, we obtain the approximate expression (4.12) for the scattering amplitude, which corresponds to the allowance for the particle Feynman paths. These paths can be considered as a classical paths and coincide in the case of the scattering of high-energy particles through small angles to straight-line paths trajectories. 


\section{Conclusions}

Asymptotic behavior of scattering amplitude for two scalar particles at high energy and fixed momentum transfers was studied. In the framework of quasi-potential approach and the modified perturbation theory the systematic scheme of finding the corrections to the principal asymptotic leading scattering amplitudes was constructed and developed. Results obtained by two different approaches (quasi potential and functional) for this problem, as it has shown that they are identical. Results obtained by us are extended to the case of scalar particles of the field $\varphi(x)$ interacting with a vector and gravitational fields. The first correction to the leading eikonal scattering amplitude in quantum field theory was obtained.

\section{Acknowledgments}

We are grateful to Profs. B.M. Barbashov, V.N.Pervushin for valuable discussions and Prof. G. Veneziano for suggesting this problem and his encouragement. N.S.H. is also indebted to Prof. H. Fried for reading the manuscript and making useful remarks for improvements. This work was supported in part by the International Center for Theoretical Physics, Trieste, the Abdus Salam International Atomic Energy Agency, the United Nations Educational, Scientific and Cultural Organization, by a grand TRIGA and by the Vietnam National University under Contract QG.TD.10.02.

\section{A The kernel of the quasi-potential equation [25]}

We denote by $G\left(\mathbf{p}, \mathbf{p}^{\prime}, \varepsilon_{\mathbf{p}}, \varepsilon_{\mathbf{q}}, \mathbf{E}\right)$ the total Green function for two particles, where $\mathbf{p}$ and $\mathbf{p}^{\prime}$ are the momenta of the initial and final states in c.m.s and $2 E=\sqrt{s}$ is the total energy.

In these notations the Bethe-Salpeter equation is of the form

$$
\begin{aligned}
& G\left(\mathbf{p}, \mathbf{p}^{\prime}, \varepsilon_{\mathbf{p}}, \varepsilon_{\mathbf{p}^{\prime}}, \mathbf{E}\right)=\mathbf{i} \mathbf{F}\left(\mathbf{p}, \varepsilon_{\mathbf{p}}, \mathbf{E}\right) \delta\left(\mathbf{p}-\mathbf{p}^{\prime}\right) \delta\left(\varepsilon_{\mathbf{p}}-\varepsilon_{\mathbf{p}^{\prime}}\right) \\
& +F\left(\mathbf{p}, \varepsilon_{\mathbf{p}}, \mathbf{E}\right) \int \mathbf{K}\left(\mathbf{p}, \mathbf{q}, \varepsilon_{\mathbf{p}}, \varepsilon, \mathbf{E}\right) \mathbf{G}\left(\mathbf{q}, \mathbf{p}^{\prime}, \varepsilon, \varepsilon_{\mathbf{p}^{\prime}}, \mathbf{E}\right) \mathbf{d} \mathbf{q} \mathbf{d} \varepsilon
\end{aligned}
$$

where

$$
\begin{aligned}
& i F\left(\mathbf{p}, \varepsilon_{\mathbf{p}}, \mathbf{E}\right)=\frac{\mathbf{2}}{\pi} \mathbf{D}\left(\mathbf{E}+\varepsilon_{\mathbf{p}}, \mathbf{p}\right) \mathbf{D}\left(\mathbf{E}-\varepsilon_{\mathbf{p}}, \mathbf{p}\right) \\
& D\left(E+\varepsilon_{p}, \mathbf{p}\right)=\frac{\mathbf{1}}{\left(\mathbf{E}+\varepsilon_{\mathbf{p}}\right)^{\mathbf{2}}-\mathbf{p}^{\mathbf{2}}-\mathbf{m}^{\mathbf{2}}+\mathbf{i} \epsilon} .
\end{aligned}
$$

Now we introduce formally the scattering amplitude $T$ which on the mass - shell $\varepsilon_{p}=\varepsilon_{p^{\prime}}=$ $0, \quad p^{2}=p^{\prime 2}=E^{2}-m^{2}$ gives the physical scattering amplitude:

$$
\begin{gathered}
G\left(\mathbf{p}, \mathbf{p}^{\prime}, \varepsilon_{\mathbf{p}}, \varepsilon_{\mathbf{p}^{\prime}}, \mathbf{E}\right)-\mathbf{i} \mathbf{F}\left(\mathbf{p}, \varepsilon_{\mathbf{p}}, \mathbf{E}\right) \delta\left(\mathbf{p}-\mathbf{p}^{\prime}\right) \delta\left(\varepsilon_{\mathbf{p}}-\varepsilon_{\mathbf{p}^{\prime}}\right)= \\
=i F\left(\mathbf{p}, \varepsilon_{\mathbf{p}}, \mathbf{E}\right) \mathbf{T}\left(\mathbf{p}, \mathbf{p}^{\prime}, \varepsilon_{\mathbf{p}}, \varepsilon_{\mathbf{p}^{\prime}}, \mathbf{E}\right) \mathbf{F}\left(\mathbf{p}^{\prime}, \varepsilon_{\mathbf{p}^{\prime}}, \mathbf{E}\right) .
\end{gathered}
$$


Then inserting (A.3) into $(A .1)$, we get for $T$ the equation

$$
\begin{gathered}
T\left(\mathbf{p}, \mathbf{p}^{\prime}, \varepsilon_{\mathbf{p}}, \varepsilon_{\mathbf{p}^{\prime}}, \mathbf{E}\right)=\mathbf{K}\left(\mathbf{p}, \mathbf{p}^{\prime}, \varepsilon_{\mathbf{p}}, \varepsilon_{\mathbf{p}^{\prime}}, \mathbf{E}\right) \\
+\int d \mathbf{q} \mathbf{d} \varepsilon \mathbf{K}\left(\mathbf{p}, \mathbf{q}, \varepsilon_{\mathbf{p}}, \varepsilon, \mathbf{E}\right) \mathbf{F}(\mathbf{q}, \varepsilon, \mathbf{E}) \mathbf{T}\left(\mathbf{q}, \mathbf{p}^{\prime}, \varepsilon, \varepsilon_{\mathbf{p}^{\prime}}, \mathbf{E}\right) .
\end{gathered}
$$

We wish to obtain an equation of the Lippmann - Schwinger type for a certain function $T\left(\mathbf{p}, \mathbf{p}^{\prime}, \mathbf{E}\right)$ which on the mass-shell $p^{2}=p^{\prime 2}=E^{2}-m^{2}$ would give the physical scattering amplitude:

$$
T\left(\mathbf{p}, \mathbf{p}^{\prime}, \mathbf{E}\right)=\mathbf{V}\left(\mathbf{p}, \mathbf{p}^{\prime}, \mathbf{E}\right)+\int \mathbf{d q} \mathbf{V}(\mathbf{p}, \mathbf{q}, \mathbf{E}) \mathbf{F}(\mathbf{q}, \mathbf{E}) \mathbf{T}\left(\mathbf{q}, \mathbf{p}^{\prime}, \mathbf{E}\right),
$$

where

$$
\begin{aligned}
F(\mathbf{q}, \mathbf{E})=\int \mathbf{d} \varepsilon \mathbf{F}(\mathbf{q}, \varepsilon, \mathbf{E}) & =-\frac{\mathbf{2 i}}{\pi} \int \mathbf{d} \varepsilon \frac{\mathbf{1}}{(\mathbf{E}+\varepsilon)^{\mathbf{2}}-\mathbf{p}^{\mathbf{2}}-\mathbf{m}^{\mathbf{2}}} \times \frac{\mathbf{1}}{(\mathbf{E}-\varepsilon)^{\mathbf{2}}-\mathbf{p}^{\mathbf{2}}-\mathbf{m}^{\mathbf{2}}} \\
& =\frac{1}{\sqrt{q^{2}+m^{2}}\left(q^{2}+m^{2}-E^{2}\right)} .
\end{aligned}
$$

On the mass-shell, the total energy $E=\frac{\sqrt{s}}{2}$, we receive the kenel that is brought out in Eq.(2.1)

$$
K\left(q^{2} ; s\right) \equiv F\left(q, E=\frac{\sqrt{s}}{2}\right)=\frac{1}{\sqrt{q^{2}+m^{2}}\left(q^{2}+m^{2}-\frac{s}{4}\right)} .
$$

This can be achieved by a conventional choice of the potential $V\left(\mathbf{p}, \mathbf{p}^{\prime}, \mathbf{E}\right)$, which can obviously be made by different methods. There are two methods that have been suggested for constructing a complex potential dependent on energy with the help of which one can obtain from an equation of the Schrödinger type the exact scattering amplitude on the mass - shell.

The first method is based on the two-time Green function [23] which in the momentum space is defined

$$
G\left(\mathbf{p}, \mathbf{p}^{\prime}, \mathbf{E}\right)=\int \mathbf{d} \varepsilon_{\mathbf{p}} \mathbf{d} \varepsilon_{\mathbf{p}^{\prime}} \mathbf{G}\left(\mathbf{p}, \mathbf{p}^{\prime}, \varepsilon_{\mathbf{p}}, \varepsilon_{\mathbf{p}^{\prime}}, \mathbf{E}\right) .
$$

Then using $(A .3)$ and $(A .8)$ we can determine the corresponding off-shell scattering amplitude

$$
T_{1}\left(\mathbf{p}, \mathbf{p}^{\prime}, \mathbf{E}\right)=\frac{1}{\mathbf{F}(\mathbf{p}, \mathbf{E}) \mathbf{F}\left(\mathbf{p}^{\prime}, \mathbf{E}\right)} \int \mathbf{F}\left(\mathbf{p}, \varepsilon_{\mathbf{p}}, \mathbf{E}\right) \mathbf{T}\left(\mathbf{p}, \mathbf{p}^{\prime}, \varepsilon_{\mathbf{p}}, \varepsilon_{\mathbf{p}^{\prime}}, \mathbf{E}\right) \mathbf{F}\left(\mathbf{p}^{\prime}, \varepsilon_{\mathbf{p}^{\prime}}, \mathbf{E}\right) \mathbf{d} \varepsilon_{\mathbf{p}} \mathbf{d} \varepsilon_{\mathbf{p}^{\prime}}
$$

From expression $(A .9)$ it is directly seen that $T$ on mass-shell $p^{2}=p^{\prime 2}=E^{2}-m^{2}$ coincides with the scattering amplitude $T\left(\mathbf{p}, \mathbf{p}^{\prime}, \mathbf{0}, \mathbf{0}, \mathbf{E}\right) \equiv \mathbf{T}\left(\mathbf{p}, \mathbf{p}^{\prime}, \mathbf{E}\right)$. The potential $V_{1}$ for Eq.(A.5) is constructed by iteration of Eqs. $(A .4),(A .5)$ and $(A .9)$. In particular, in the lowest order, we have

$$
V_{1}\left(\mathbf{p}, \mathbf{p}^{\prime}, \mathbf{E}\right)=\frac{1}{\mathbf{F}(\mathbf{p}, \mathbf{E}) \mathbf{F}\left(\mathbf{p}^{\prime}, \mathbf{E}\right)} \int \mathbf{F}\left(\mathbf{p}, \varepsilon_{\mathbf{p}}, \mathbf{E}\right) \mathbf{K}\left(\mathbf{p}, \mathbf{p}^{\prime}, \varepsilon_{\mathbf{p}}, \varepsilon_{\mathbf{p}^{\prime}}, \mathbf{E}\right) \mathbf{F}\left(\mathbf{p}^{\prime}, \varepsilon_{\mathbf{p}^{\prime}}, \mathbf{E}\right) \mathbf{d} \varepsilon_{\mathbf{p}} \mathbf{d} \varepsilon_{\mathbf{p}^{\prime}}
$$


The second method consists in constructing the potential $V_{2}$ for Eq.(A.4) by means of the scattering amplitude $\mathrm{T}$ on the mass-shell obtained by perturbation theory, e.g. from Eq.(A.4) and the iterations of Eq.(A.5) accompanied by the transition to the mass-shell.

We write down Eq.(A.5) in the symbolic form $T_{2}=V_{2}+V_{2} \times T_{2}$ and obtain in the lowest orders of $V_{2}$ the expressions

$$
\begin{gathered}
V_{2}^{(2)}=\left[T^{(2)}\right], V^{(4)}=\left[T^{(4)}\right]-\left[V_{2}^{(2)} \times T_{2}^{(2)}\right], \\
V_{2}^{(6)}=\left[T^{(6)}\right]-\left[V_{2}^{(2)} \times T_{2}^{(4)}\right]-\left[V_{2}^{(4)} \times T_{2}^{(2)}\right] \ldots,
\end{gathered}
$$

where the square brackets mean here the transition to the mass-shell. Hence, it follows that in the second method we get a local potential dependent only on $\left(\mathbf{p}-\mathbf{p}^{\prime}\right)^{2}$ and $E$ and in r-space on r and $E$.

We shall consider, as an example, the application of the above methods to a model of quantum field theory, in which scalar particles of mass m interact by exchanging scalar "photons" of small mass $\mu$. We shall put $\mu=0$ where it is possible. In the ladder approximation (without considering the crossing symmetry) the kernel of Eq.(A.5) is of the form

$$
K\left(\mathbf{p}, \mathbf{p}^{\prime}, \varepsilon_{\mathbf{p}}, \varepsilon_{\mathbf{p}^{\prime}}, \mathbf{E}\right)=-\frac{\mathbf{i g}^{2}}{(2 \pi)^{4}} \frac{1}{\left(\varepsilon_{\mathbf{p}}-\varepsilon_{\mathbf{p}^{\prime}}\right)^{2}-\left(\mathbf{p}-\mathbf{p}^{\prime}\right)^{2}-\mu^{2}+\mathbf{i} \epsilon}
$$

\section{B Some integrals used in this paper}

We consider the integral

$$
I_{1}=\int_{-\infty}^{\infty} d z V\left(\sqrt{\mathbf{r}_{\perp}^{2}+z^{2}} ; s\right)=-\frac{g^{2}}{8 \pi s} \int_{-\infty}^{\infty} d z \frac{e^{-\mu\left(\sqrt{\mathbf{r}_{\perp}^{2}+z^{2}}\right)}}{\sqrt{\mathbf{r}_{\perp}^{2}+z^{2}}}
$$

we have

$$
\begin{gathered}
I_{1}=-\frac{g^{2}}{4(2 \pi)^{4} s} \int d^{3} p \int_{-\infty}^{+\infty} d z \frac{e^{i \mathbf{p r}}}{\mu^{2}+p^{2}}=-\frac{g^{2}}{4(2 \pi)^{4} s} \int d^{3} p \int_{-\infty}^{+\infty} d z \frac{e^{i\left(p_{\perp} r_{\perp}+p_{/ /} z\right)}}{\mu^{2}+p^{2}} \\
=-\frac{g^{2}}{4(2 \pi)^{4} s} \int d^{3} p \frac{e^{i\left(p_{\perp} r_{\perp}\right)}}{\mu^{2}+p^{2}} \int_{-\infty}^{+\infty} d z e^{i p / / z} \\
=-\frac{g^{2}}{4(2 \pi)^{4} s} \int d^{2} p_{\perp} d p / / \frac{e^{i\left(p_{\perp} r_{\perp}\right)}}{\mu^{2}+p_{\perp}^{2}+p_{/ /}^{2}} \times(2 \pi) \delta\left(p_{/ /}\right) \\
=-\frac{g^{2}}{4(2 \pi)^{3} s} \int d^{2} p_{\perp} e^{i\left(p_{\perp} r_{\perp}\right)} \int d p_{/ /} \frac{\delta\left(p_{/ /}\right)}{\mu^{2}+p_{\perp}^{2}+p_{/ /}^{2}} \\
=-\frac{g^{2}}{4(2 \pi)^{3} s} \int d^{2} p_{\perp} \frac{e^{i\left(p_{\perp} r_{\perp}\right)}}{\mu^{2}+p_{\perp}^{2}}=-\frac{g^{2}}{4(2 \pi)^{2} s} K_{0}\left(\mu\left|r_{\perp}\right|\right),
\end{gathered}
$$


with $K_{0}\left(\mu\left|r_{\perp}\right|\right)=\frac{1}{2 \pi} \int d^{2} p_{\perp} \frac{e^{i\left(p_{\perp} r_{\perp}\right)}}{\mu^{2}+p_{\perp}^{2}}$ is the MacDonald function of zeroth order.

The integral

$$
I_{2}=\int d^{2} r_{\perp} e^{i \Delta_{\perp} r_{\perp}} K_{0}\left(\mu\left|r_{\perp}\right|\right)=(2 \pi) \int d\left|r_{\perp}\right|\left|r_{\perp}\right| J_{(0)}\left(\Delta_{\perp}\left|r_{\perp}\right|\right) K_{0}\left(\mu\left|r_{\perp}\right|\right)=\frac{2 \pi}{\mu^{2}-t} .
$$

The integral

$$
\begin{gathered}
I_{3}=\int d^{2} r_{\perp} e^{i \Delta_{\perp} r_{\perp}} K_{0}^{2}\left(\mu\left|r_{\perp}\right|\right)=\int d^{2} r_{\perp} e^{i \Delta_{\perp} r_{\perp}}\left(\frac{1}{2 \pi} \int d^{2} q \frac{e^{i \mathbf{q} \mathbf{r}_{\perp}}}{q^{2}+\mu^{2}}\right) K_{0}\left(\mu\left|r_{\perp}\right|\right) \\
=\frac{1}{2 \pi} \int d^{2} q \frac{1}{q^{2}+\mu^{2}} \int d^{2} r_{\perp} e^{i\left(q+\Delta_{\perp}\right) r_{\perp}} K_{0}\left(\mu\left|r_{\perp}\right|\right) \\
=\frac{1}{2 \pi}(2 \pi) \int d^{2} q \frac{1}{q^{2}+\mu^{2}} \frac{1}{\left(q+\Delta_{\perp}\right)^{2}+\mu^{2}}
\end{gathered}
$$

here, the result of the integral that obtained from calculating $I_{2}$ have been used.

Using method of Feynman parameter integral $\frac{1}{a b}=\int_{0}^{1} \frac{d x}{[a x+b(1-x)]^{2}}$, we have

$$
\begin{gathered}
I_{3}=\int_{0}^{1} d x \int d^{2} q \times \frac{1}{\left\{\left(q^{2}+\mu^{2}\right) x+\left[\left(q+\Delta_{\perp}\right)^{2}+\mu^{2}\right](1-x)\right\}^{2}} \\
=\int_{0}^{1} d x \int d^{2} q \frac{1}{\left[q^{2}+2 q \Delta_{\perp}(1-x)+\Delta_{\perp}^{2}(1-x)+\mu^{2}\right]^{2}} \\
=\int_{0}^{1} d x \frac{i(-\pi) \Gamma(1)}{\left[\Delta_{\perp}^{2}(1-x)+\mu^{2}-\Delta_{\perp}^{2}(1-x)^{2}\right] \Gamma(2)} \\
=(-i \pi) \int_{0}^{1} d x \frac{1}{\left[\mu^{2}+\Delta_{\perp}^{2} x(1-x)\right]}=(-i \pi) \int_{0}^{1} \frac{d x}{\left[\mu^{2}-t x(1-x)\right]}= \\
=(-i \pi) \times \frac{1}{t \sqrt{1-\frac{4 \mu^{2}}{t}}} \ln \frac{1-\sqrt{1-\frac{4 \mu^{2}}{t}}}{1+\sqrt{1-\frac{4 \mu^{2}}{t}}} \equiv(-i \pi) \times F_{1}(t) .
\end{gathered}
$$

Finally, we calculate the integral

$$
\begin{gathered}
I_{4}=\int d^{2} r_{\perp} e^{i \Delta_{\perp} r_{\perp}} K_{0}^{3}\left(\mu\left|r_{\perp}\right|\right) \\
=\int d^{2} r_{\perp} e^{i \Delta_{\perp} r_{\perp}}\left(\frac{1}{2 \pi} \int d^{2} q_{1} \frac{e^{i q_{1} r_{\perp}}}{q_{1}^{2}+\mu^{2}}\right) \times\left(\frac{1}{2 \pi} \int d^{2} q_{2} \frac{e^{i q_{2} r_{\perp}}}{q_{2}^{2}+\mu^{2}}\right) K_{0}\left(\mu\left|r_{\perp}\right|\right) \\
=\frac{1}{(2 \pi)^{2}} \int \frac{d^{2} q_{1} d^{2} q_{2}}{\left(q_{1}^{2}+\mu^{2}\right)\left(q_{2}^{2}+\mu^{2}\right)} \times \int d^{2} x_{\perp} \exp \left[i\left(q_{1}+q_{2}+\Delta_{\perp}\right) x_{\perp}\right] K_{0}\left(\mu\left|r_{\perp}\right|\right) \\
=\frac{1}{(2 \pi)^{2}} \int d^{2} q_{1} d^{2} q_{2} \times \frac{1}{\left(q_{1}^{2}+\mu^{2}\right)\left(q_{2}^{2}+\mu^{2}\right)\left[\left(q_{1}+q_{2}+\Delta_{\perp}\right)^{2}+\mu^{2}\right]} .
\end{gathered}
$$

Apply the result that we obtained when calculating $I_{3}$ to this integral, we derive 


$$
\begin{aligned}
& \int d^{2} q_{1} \frac{1}{\left(q_{1}^{2}+\mu^{2}\right)\left[\left(q_{1}+q_{2}+\Delta_{\perp}\right)^{2}+\mu^{2}\right]} \\
= & (-i \pi) \int_{0}^{1} d x \frac{1}{\left[\mu^{2}+\left(q_{2}+\Delta_{\perp}\right)^{2} x(1-x)\right]},
\end{aligned}
$$

so

$$
I_{4}=\frac{1}{(2 \pi)^{2}}(-i \pi) \int_{0}^{1} d x \int d^{2} q_{2} \times \frac{1}{\left(q_{2}^{2}+\mu^{2}\right)\left[\mu^{2}+\left(q_{2}+\Delta_{\perp}\right)^{2} x(1-x)\right]}
$$

From method of Feynman parameter integral, again, we obtain

$$
\begin{gathered}
I_{4}=-\frac{i}{(4 \pi)} \int_{0}^{1} \frac{d x}{x(1-x)} \int_{0}^{1} d y \int d^{2} q_{2} \times \\
\times \frac{1}{\left\{\left[\left(q_{2}+\Delta_{\perp}\right)^{2}+B\right] y+\left(q_{2}^{2}+\mu^{2}\right)(1-y)\right\}^{2}} \\
=-\frac{i}{(4 \pi)} \int_{0}^{1} \frac{d x}{x(1-x)} \int_{0}^{1} d y \int d^{2} q_{2} \frac{1}{\left(q_{2}^{2}+2 q_{2} \Delta_{\perp} y+C\right)^{2}} \\
=-\frac{i}{(4 \pi)}(-i \pi) \int_{0}^{1} \frac{d x}{x(1-x)} \int_{0}^{1} d y \frac{1}{\left[C-\left(\Delta_{\perp} y\right)^{2}\right]} \\
\left.=-\frac{1}{4} \int_{0}^{1} \frac{d x}{x(1-x)} \int_{0}^{1} d y \frac{1}{\left[C-\left(\Delta_{\perp} y\right)^{2}\right]} .\right)
\end{gathered}
$$

where

$$
B=\frac{\mu^{2}}{x(1-x)}, C=\left(\Delta_{\perp}^{2}+B\right) y+\mu^{2}(1-y)=\left[\frac{\mu^{2}}{x(1-x)}-t\right] y+\mu^{2}(1-y)
$$

then

$$
\begin{gathered}
I_{4}=-\frac{1}{4} \int_{0}^{1} \frac{d x}{x(1-x)} \int_{0}^{1} d y \times \frac{1}{\left[\frac{\mu^{2}}{x(1-x)}-t\right] y+\mu^{2}(1-y)+t y^{2}} \\
=-\frac{1}{4} \int_{0}^{1} d y \int_{0}^{1} d x \frac{1}{-(1-y)\left(t y-\mu^{2}\right) x(1-x)+\mu^{2}}=-\frac{1}{4} \int_{0}^{1} d y \int_{0}^{1} d x \frac{1}{D x^{2}-D x+\mu^{2}} \\
=-\frac{1}{4} \int_{0}^{1} \frac{d y}{D} \int_{0}^{1} \frac{d x}{x^{2}-x+\frac{\mu^{2}}{D}}=-\frac{1}{4} \int_{0}^{1} \frac{d y}{D} \int_{0}^{1} \frac{d x}{\left(x-x_{1}\right)\left(x-x_{2}\right)} \\
=-\frac{1}{4} \int_{0}^{1} \frac{d y}{D} \frac{1}{x_{1}-x_{2}} \ln \left|\frac{\left(1-x_{1}\right) x_{2}}{\left(1-x_{2}\right) x_{1}}\right|
\end{gathered}
$$

here, $D=-(1-y)\left(t y-\mu^{2}\right)=t y^{2}+\left(\mu^{2}-t\right) y+\mu^{2}$ and $x_{1} ; x_{2}$ are roots of equation $x^{2}-x+\frac{\mu^{2}}{D}=0$.

Noting that: $x_{1}+x_{2}=1 \Rightarrow 1-x_{1}=x_{2} ; 1-x_{2}=x_{1}$, and:

$$
x_{1}-x_{2}=\sqrt{1-\frac{4 \mu^{2}}{D}} \approx 1-\frac{2 \mu^{2}}{D},
$$

hence 


$$
\ln \left|\frac{\left(1-x_{1}\right) x_{2}}{\left(1-x_{2}\right) x_{1}}\right|=\ln \left|\frac{x_{2}^{2}}{x_{1}^{2}}\right|=2 \ln \left|\frac{1-\sqrt{1-\frac{4 \mu^{2}}{D}}}{1+\sqrt{1-\frac{4 \mu^{2}}{D}}}\right| \approx 2 \ln \left|\frac{\mu^{2}}{D-\mu^{2}}\right|
$$

so that

$$
\begin{gathered}
I_{4}=-\frac{1}{2} \int_{0}^{1} d y \frac{1}{D-2 \mu^{2}} \ln \left|\frac{\mu^{2}}{D-\mu^{2}}\right| \\
=-\frac{1}{2} \int_{0}^{1} d y \frac{1}{\left(t y+\mu^{2}\right)(y-1)} \ln \left|\frac{\mu^{2}}{y\left(t y+\mu^{2}-t\right)}\right| \equiv-\frac{1}{2} F_{2}(t)
\end{gathered}
$$

\section{References}

[1] H. D. I.Abarbanel and C. Itzykson, Phys. Rev. Lett. 23 (1969) 53.

[2] B. M. Barbashov, S. P. Kuleshov, V. A. Matveev, V. N. Pervushin, A. N. Sissakian and A. N. Tavkhelidze, Phys. Lett. 33B (1970) 484.

[3] H.M. Fried, Functional Methods and Models in Quantum Field Theory, Cambridge, Massachusetts: MIT Press. 1972; Functional Methods and Eikonal Models, Editions Frontieres, 1990; Green's Functions and Odered Exponentials, Cambridge University Press, 2002.

[4] V. R. Garsevanishvili, V. A. Matveev, L. A. Slepchenko, and A. N. Tavkhelidze, Phys. Lett. 29B (1969) 191.

[5] G. Tikotopoulos and S. B.Treinman, Phys. Rev. 3D (1971) 1037.

[6] E.Eichen and R. Jackiw, Phys. Rev. D4 (1971) 439.

[7] M. Levy and J. Sucher, Phys. Rev. 186 (1969) 1656; D2 (1971) 1716.

[8] L. N. Lipatov, Phys. Lett. B116 (1992) 411; Nucl. Phys. B365 (1991) 614.

[9] G. 'tHooft, Phys. Lett. 198B (1987) 61.

[10] D. Amati, M. Ciafaloni and G. Veneziano, Phys. Lett. 197B (1987) 81.

[11] D.Amati, M. Ciafaloni and G. Veneziano, Int. J. Mod. Phys. A3 (1988) 1615.

[12] D. Amati, M. Ciafaloni and G. Veneziano, Nucl. Phys. B347 (1990) 550.

[13] E. Verlinde and H. Verlinde, Nucl. Phys. B371 (1992) 246.

[14] D. Kabat and M. Ortiz, Nucl. Phys. B388 (1992) 570.

[15] M. Fabbrichesi, R. Pettorino, G. Veneziano, G. A. Vilkovisky, Nucl. Phys. B419 (1994) 147.

[16] I.Muzinich and M. Soldate, Phys. Rev. D37 (1988) 353. 
[17] R. Kirschner, Phys. Rev. D52, N4 (1995) 2333.

[18] Nguyen Suan Han and E. Ponna, Nuovo Cimento A, 110 (1997) 459.

[19] Nguyen Suan Han, Eur. Phys. J. C16 (2000) 547.

[20] Nguyen Suan Han, and Nguyen Nhu Xuan, Eur. Phys. J. C24 (2002) 643; gr-qc/0203054.

[21] V. R. Garsevanishvili, V. A. Matveev, and L. A. Slepchenko, "Fiz. Elm. Chast. At. Yadra" $1(1970) 91$.

[22] V. R. Garsevanishvili, V. A. Matveev, L. A. Slepchenko, and A. N. Tavkhelidze, Theor. Math. Fiz. 6(1971) 36.

[23] S. P. Kuleshov, V. A. Matveev, A. N. Sissakian, and M. A. Smodyrev, Theor. Math. Fiz. 14B (1973) 325.

[24] A. A. Logunov and A. N. Tavkhelidze. Nuovo Cim, 29 (1963) 380.

[25] A.A. Logunov, Nguyen Van Hieu and O.A. Khrustalev, Nucl. Phys., 50 (1964) 295.

[26] Nguyen Van Hieu and R. N. Faustov, Nucl. Phys., 53 (1964) 337.

[27] I. T. Todorov, Phys. Rev.D, 3 (1971)2351.

[28] V. G. Kadyshevskii and A. N. Tavkhelidze: Problems of Theoretical Physics (Delicated to N.N. Bogoliubov on occasion of his 60th birthday), Nauka, 1969.

[29] M. A. Mestvirshvili and G. L. Rcheulishvili, Theor. Math. Fiz. 8 (1970) 206.

[30] A. T. Filippov, Nucl. Phys., " Fiz. Elm. Chast. At. Yadra" 10 (1970) 501.

[31] E. S. Fradkin, Acta Phys. Hung 19 (1965) 175; Nucl.Phys. 76 (1966) 588.

[32] V. N. Pervushin, Theor. Math. Fiz.14(1972)332. 